\title{
Manufacturing of High Pressure Die Casting Die Inserts Using SLM
}

\author{
Roland STOLT ${ }^{\mathrm{a}, 1}$ and Anders E. W. JARFORS ${ }^{\mathrm{b}}$ \\ a Jönköping University, Dept. of Product Development, Production and Design \\ b Jönköping University, Dept. of Materials and Manufacuring
}

\begin{abstract}
Dies for high pressure die casting are normally manufactured by machining of slabs of tool steel to the required dimensions. This manufacturing requires several steps such as rough machining, heat-treatment, EDM (electro discharge machining) and polishing. With the AM (additive manufacturing) method SLM (Selective Laser Melting) it has become possible to print the dies fully or in part. Several advantages are expected, such as a better thermal distribution in the die in service and thereby extended die life and better component quality. This is due to the possibility of making the cooling channels conformal. There are also expectations of reduced time and cost in the manufacturing process due to fewer manufacturing steps and better material utilisation. Hopes are to print a net shape or near net shape that can be used directly as a die component. In this paper it is investigated to what extent this can be fulfilled by printing two die inserts to be used for casting fatigue test samples of aluminium. They were printed on a 3D Systems ProX DMP 300 in maraging steel powder. The result is that it is possible to obtain a sufficiently smooth surface die surface. However, the current design with an SLM insert fitted in a machined die makes extensive post printing manufacturing necessary.
\end{abstract}

Keywords. Additive Manufacturing; AM; High Pressure Die Casting; HPDC; Selective Laser Melting; SLM Dies; Conformal cooling channels.

\section{Introduction}

Dies and moulds are increasingly being manufactured by additive manufacturing (AM) methods such as Electron Beam melting (EBM) and Selective Laser Melting (SLM). Examples includes dies for injection moulding of thermo plastics and high pressure die casting (HPDC) of light metal alloys. Also dies for the extrusion of aluminium profiles are being manufactured by AM [1]. One of the main motivations for the AM of dies is gaining better control of the die temperature by introducing conformal cooling channels. By manufacturing the dies by AM, the cooling channels can be located at exactly the places where the cooling is needed making the temperature distribution of the die more even and thereby extending the die life due to reduced thermal stresses. Better control of the temperature also mean that the part quality can be increased, and that the cycle times can be reduced.

The conformal cooling channels are often geometrically complicated and there are few, if any other ways than AM by which they can be manufactured. In this paper, the possibilities of using the SLM process for HPDC dies with conformal cooling will be

\footnotetext{
${ }^{1}$ Corresponding Author. [roland.stolt@ju.se]
} 
explored. In the HPDC case, the solidification phase is short, so shortening the cycle time is not the primary motivation. Instead, better control of the die temperature is expected to lead to a longer tool life, better part quality and less use of spraying.

However, there are many challenges in this. Obtaining sufficiently good tolerances and surface quality of the die directly from printing is one. The current slab designs of dies are massive and ill-suited for printing. They need redesigning. The objective of this paper is to propose a useful design of printed die inserts and to find which manufacturing operations in addition to the printing that will be necessary to arrive at the needed surfaces and tolerances. This is done by conducting an example, where a traditionally manufactured HPDC die used for producing fatigue test samples is converted to version with a machined base fitted with SLM inserts. In the paper, it is accounted for how the inserts were designed and how the whole manufacturing process was carried out. The result was a complete specification and manufactured die set ready for production of fatigue test samples.

\section{Literature}

AM has its own set of rules on how to make the geometry suitable for printing. This is often referred to as Design for Additive Manufacturing (DFAM) [2-6].

Unlike other manufacturing processes, the part can be arbitrarily complex without adding to the part cost. Instead, when designing for AM, the main objectives are to minimise the volume of the part and avoid overhangs. The combination of the low sensitivity to complexity and the requirement to minimise weight makes it very useful to use topology optimisation, creating light but complex structures $[7,8]$.

AM has also found use in the manufacturing of moulds and dies. This is well established in injection moulding. Since injection moulding operates with much lower temperature than in HPDC, moulds can be designed with thinner walls than HPDC. This gives a low material volume in the mould which is beneficial for SLM manufacture. Since the efficient cooling of the mould has a direct impact on the cycle time much effort has been made in research to optimize the location and shape of the cooling channels in moulds [9-12]. A suggestion has been made on further improving the cooling efficiency by letting the coolant flow through printed porous structures [13]. One practical example of an SLM printed mould is found on the web page [14]. This is a complete mould that required very few secondary manufacturing operations. It was possible to make several thousand parts in ABS plastic from it without any visible wear.

The challenges in HPDC are far greater because the high pressure and temperature makes it necessary to increase the wall thickness, making them difficult and expensive to print. Other challenges are that the size of HPDC dies are considerable, often exceeding the printing envelopes of SLM printers. This makes it necessary to fit a printed insert in a machined die base. However, it has been shown [15] that most of the manufacturing cost, around $30 \%$ is invested in the die inserts. This shows that there is opportunity for putting effort into the printing. It needs to be investigated in absolute numbers what the cost effect will be by switching from traditional dies to partly of fully printing them.

Printing the inserts will mean that die-base can be a less complicated, not requiring hardening and electro discharge machining (EDM). However, other challenges will emerge such as creating a sealing between conform and strait drilled cooling channels [16]. 
There are currently not many examples of printed HPDC dies found in literature. One pioneering study is [17] where the thermal distribution and structural stiffness of an imagined light weigh truss die is analysed using FEA. It was found that energy can be expected to be saved by reducing the thermal mass of the die. Further, the stiffness is expected be lower than in a conventional die. SLM as a manufacturing method was not mentioned in the study, but the proposed structure resembles the lattice structures often found in SLM geometries.

\section{Methodology}

This paper has been written as part of a three-year research project with a duration between the years 2017 and 2020. One part of the project involved examining the possibilities of SLM printing of HPDC dies. As concluded in the literature section, there are currently very few papers published on the topic, why the chosen way forward was conducting a practical case on SLM insert design and manufacturing. Assumptions were made to complete the inserts. The inserts will later be operated in actual HPDC production of aluminium samples for fatigue testing. From the production, data on the performance of the SLM die will be obtained. This will make it possible to evaluate the assumptions made. The data is expected to include the temperature distribution on the die surface to study the effect of adding the conform cooling channels. The wear on the die surface will be measured. The performance of the die surface such as sticking of aluminium and formation of cracks will be studied.

\section{Design and manufacturing procedure}

The manufactured assembly is seen in figure 1 . It consists of machined dies fitted with printed inserts. The dimensions of the dies are approximately $420 \times 270 \times 70 \mathrm{~mm}$ and of the inserts $240 \times 64 \times 35 \mathrm{~mm}$. The dies were too massive to be printed on the ProX 300DMP printer with a build envelope of $250 \times 250 \times 350 \mathrm{~mm}$. After making the inserts lighter by the removal of material and retaining a minimum of $6 \mathrm{~mm}$ wall thickness, the inserts could be printed in maraging steel $18 \mathrm{Ni300/1.2709}$. The printed inserts have been fitted in larger dies manufactured in non-heat treated tool steel 1.2312 (Holdax ${ }^{\circledR}$ ). The dies were manufactured by $\mathrm{CNC}$ machining and has straight drilled cooling channels. The assembly of dies and inserts for cover and ejector dies are seen in the figure 1. It is difficult to make a pressure tight connection between the drilled channels of the die and the conformal channels of the inserts. Instead two separate circuits were created. This is described in [16].

The fitting between inserts and dies is ISO tolerance grade $6 / 7$. 


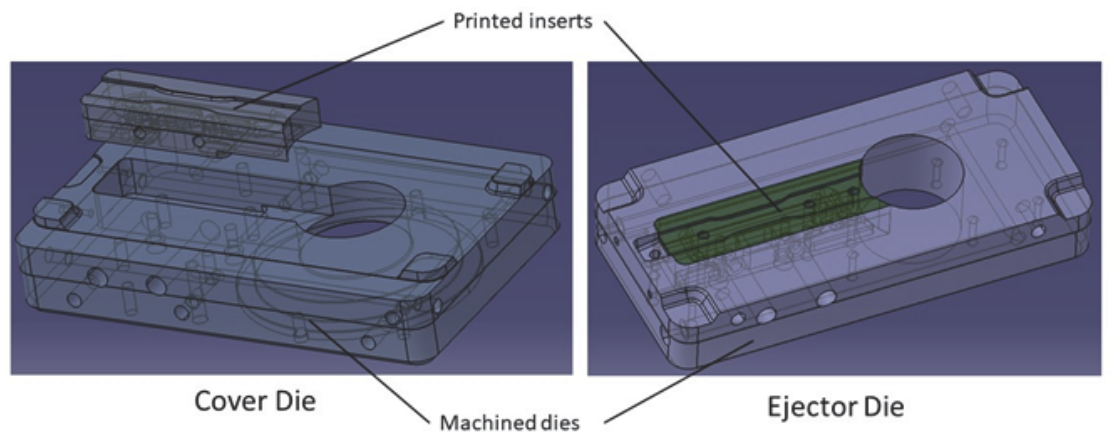

Figure1. Assembly of dies and inserts.

A picture of the two printed inserts is shown in figure 2. In the left of the figure, the side of the inserts facing the metal melt is shown (the form cavities). At the righthand side, the opposite side is shown. Note that on the opposite side, material have been removed from the inserts in order to minimize the amount of material in them. Both inserts have conformal cooling channels. Figure 3 show the $\varnothing 12 \mathrm{~mm}$ cooling channel location inside the cover die insert.

The printing time for the two inserts was 50 hours on the ProX300 printer. The cost of running the printer is roughly 120 USD per hour, so an estimate of the cost of printing is $6000 \mathrm{USD}$. The printer settings prepared in the software $3 \mathrm{dXpert}$ were: $30 \%$ laser power, $1200 \mathrm{~mm} /$ second scan speed, $0 \mathrm{~mm}$ defocus, $57 \mu \mathrm{m}$ hatch spacing and hexagonal cells of size $50 \mathrm{~mm}$ with direction inside-out.

There was a considerable time put into preparing the print and especially in removing the support structures. This was done by sawing of the inserts from the build plate and then removing the remaining support structures using chisel and pliers. The estimated time for preparation and support structure removal was around four hours for one person.
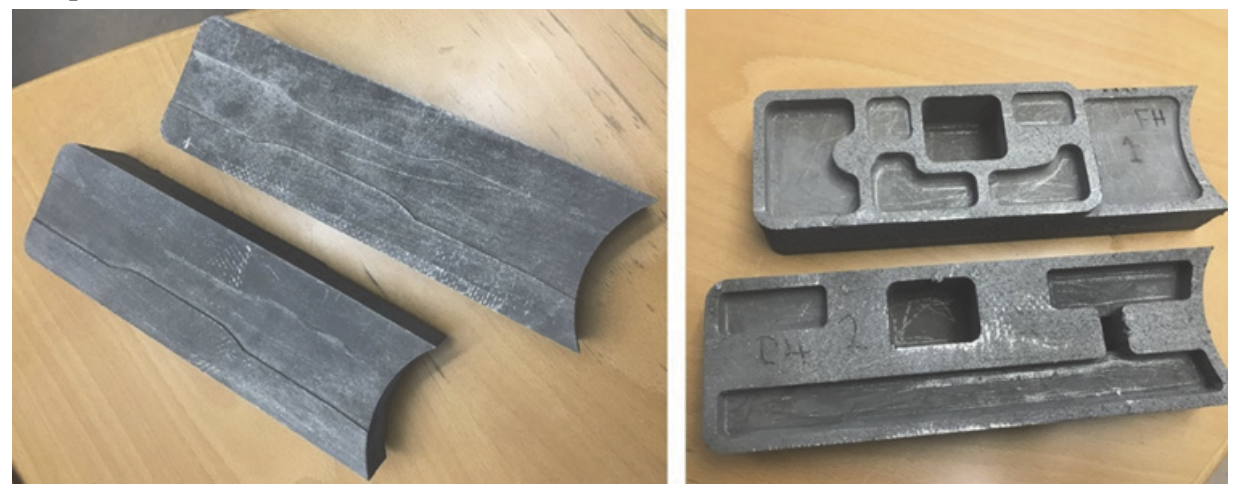

Figure2. Inserts as printed after support structure removal. 


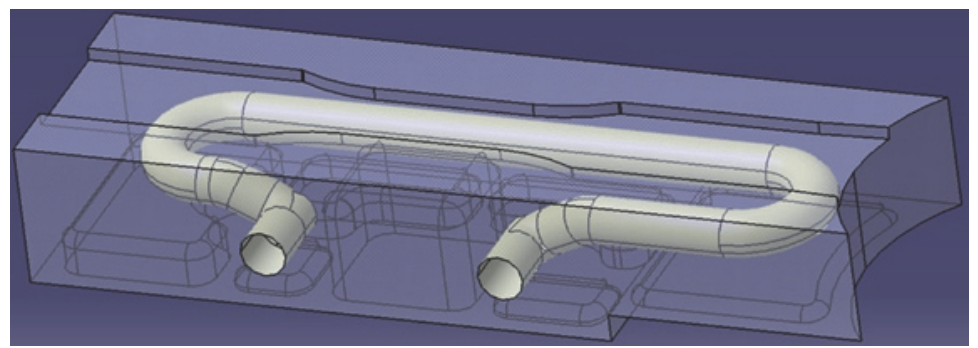

Figure3. Conformal cooling channel in the cover die insert.

Due to the dimensional precision and the surface quality of the as printed part, machining had to be conducted. The printed parts of figure 2 can be seen as "blanks" without screw attachments and holes for the ejector pins. These are added by machining. This also included several machining operations to obtain reference elements and the correct tolerances and surfaces. The inserts were age hardened to 53HRC. The ten different operations are seen in the list of operations in table 1. Refer also to figure 4 , showing the locations of the operations on the ejector die insert. The cover die was processed similarly, but without operations 5 and 10

Table 1. List of operations.

\begin{tabular}{|r|r|c|}
\hline \multicolumn{1}{|l|}{ OP } & Description of operations & Equipment \\
\hline 1 & Rough machining of bottom surface as positioning reference & CNC \\
\hline 2 & $2 \times$ M10 thread for insert fastening and positioning reference & CNC \\
\hline 3 & Rough machining of die cavity and top surface & CNC \\
\hline 4 & Rough machining fitting surface & Drill \\
\hline 6 & Ax drilled precursors for WEDM of ejector pin guides & induction \\
\hline 7 & Fine machining of fitting surfaces & CNC \\
\hline 8 & Fine machining cavity and top surface & CNC \\
\hline 9 & WEDM of ejector pin guides. & WEDM \\
\hline 10 & &
\end{tabular}

The precision on dimension, straightness and roundness on the ejector pin guides are strict. They were manufactured by first drilling holes and, after hardening, created by Wire Electro Discharge Machining (WEDM).

$(4,8)$

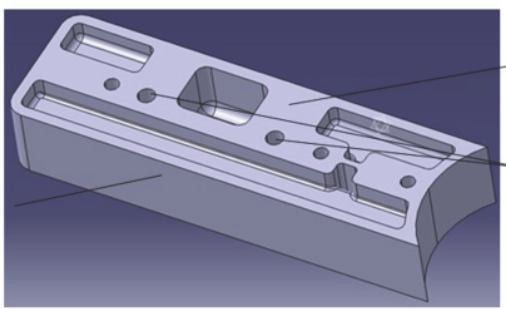

$(1)$

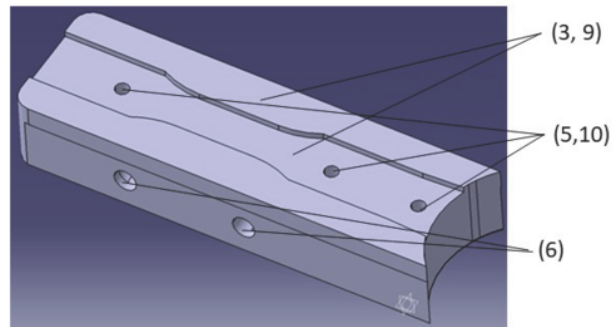

Figure4. Locations of the manufacturing operations. 
Two sets of inserts were printed. However, the first set had to be scrapped since the CAD-models had not been compensated for shrinkage and ended up having undersized dimensions. The reason for this mistake was that a number of 125 10x10x10mm samples had been printed in the same material with varying laser power, scanning speed, hatch spacing. This was done to establish a parameter window for maximizing the density using design of experiments. The cubes were arranged on the build plate as shown in figure 5 using a rectangular scan pattern.

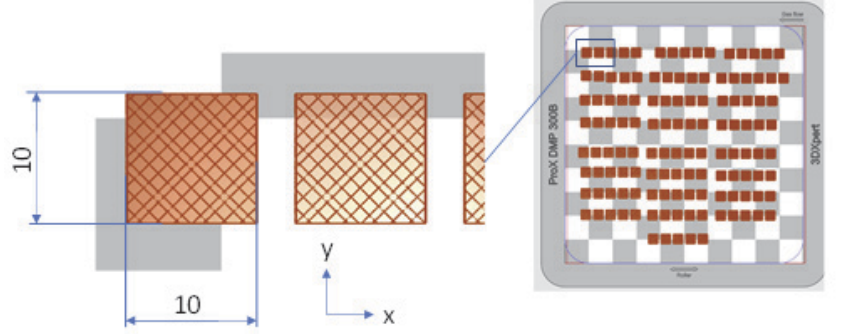

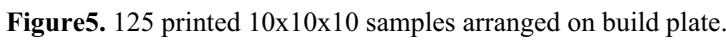

All samples except five showed a slightly oversized dimensions in the $\mathrm{x}$ and $\mathrm{y}$ directions. The five samples with undersized dimensions had been printed with parameters that were not going to be used in the insert print. The average dimensions of all samples are shown in table 2 .

Table 2. Average dimensions.

\begin{tabular}{|c|c|c|}
\hline Samples avg. & $\mathrm{X}$ & $\mathrm{Y}$ \\
\hline 1 to 25 & 10,01583 & 10,027 \\
\hline
\end{tabular}

As seen, oversized dimensions of on average $0,15 \%$ and $0,3 \%$ were obtained on the samples. This led to the erroneous conclusion that no shrinkage compensation was needed for the material. It was assumed that the inserts could be printed to nominal dimensions and that this would result in a small allowance for grinding to the correct dimensions also assuming that they would have the correct shape. However, the prints ended up with undersized dimensions and were consequently scrapped. The nominal dimensions and the dimensional outcome of the as printed cover die insert is shown in figure 6 .

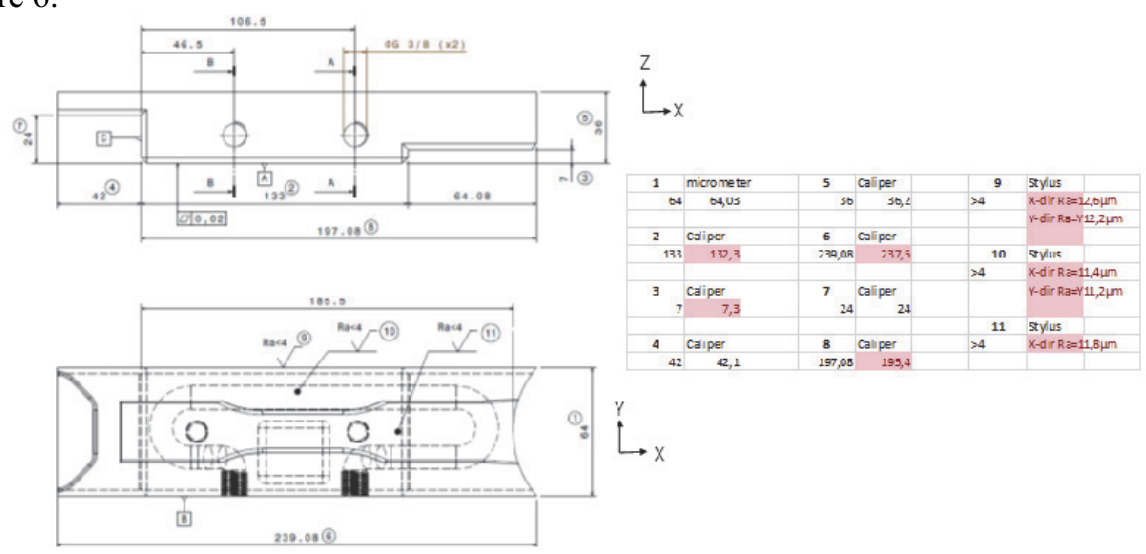

Figure6. Resulting dimensions as printed of cover die insert (scrapped). 
The inspection protocol on the right-hand side of the figure shows that, dimensions especially in the x-direction became too short. As an example, dimension 6 had a nominal length of 239,08 but was measured to 237,5 . That is more than $1,5 \mathrm{~mm}$ too short. Dimensions in $\mathrm{y}$ and $\mathrm{z}$ seem to be close to nominal or with some oversized dimension. In addition to the dimensional errors, there was also a deviation concerning the shape. Both inserts were thicker in the middle than in the ends. This was noticed by placing them on a diabase measuring table with the reference surface B (see figure 6) facing down. A rocking of about $0,05 \mathrm{~mm}$ could be detected.

The second set was printed with a uniform $+2 \%$ shrinkage compensation. This resulted in enough allowance for machining. The measured dimensions as printed are shown in the figure 7 . The drawing views in the figure shows the nominal dimensions.

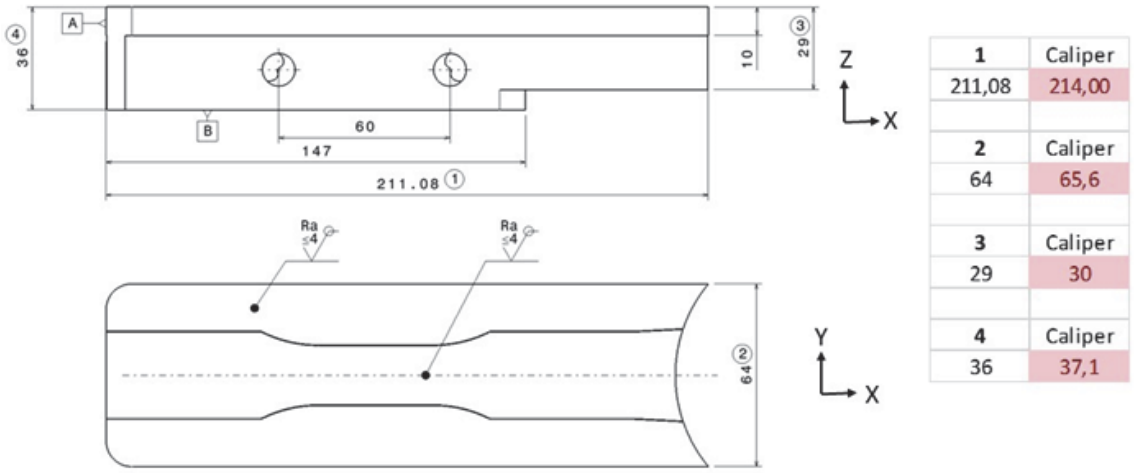

Figure7. Resulting dimensions as printed of cover die insert (scrapped).

As seen, the result of the shrinkage compensation was an allowance for machining enough to compensate for the shape and dimensional deviations. A good fit between inserts and dies could be created. The play is expected to be between 0 and $49 \mu \mathrm{m}$ corresponding to a ISO H7/h6 fit. This gives a sufficiently small play to stop ingress of molten metal according to the die manufacturing firm. In the z-direction (figure 7). The tolerances of the height of the inserts (dimension 4 in figure 6) and the depth of the corresponding pocket in the die have been selected so that the insert will protrude between 0 and $10 \mu \mathrm{m}$ over the die top surface. This ensure a slight protrusion of the insert from the die meaning that the inserts will be in contact first when closing the tool. The closing pressure of the HPDC machine will then force them down to perfect alignment with the die top surface, ensuring a minimum of flash burr.

\section{Discussion}

In the case conducted, many manufacturing operations where required after the printing. In fact, machining was required on all external surfaces. It would perhaps have been possible to use the form cavity as printed with some light post-treatment since the as printed surface roughness was around $\mathrm{Ra}=12 \mu \mathrm{m}$ as seen in the measuring protocol of figure 6 . With some hand grinding with fine emery cloth, grain 400, it was possible to decrease the Ra value to about $8 \mu \mathrm{m}$. This could possibly have been directly usable as a die surface, at least for short series (a few hundreds) of fatigue test samples. However, the compensation for the shrink was crudely done by just adding $2 \%$ 
uniformly, the decision was taken to machine also the form cavity to ensure good alignment of the cavities of the two dies.

There are more accurate ways of compensating for material shrinkage. Simulation of the build process allow the prediction of geometrical deviations so that a nonuniform compensation can be employed to counteract these effects. This also include adjusting the thermal conductivity of the support structure to avoid shrinkage and warping of printed part. The dimensional change as an effect of hardening must also be compensated. In the presented case, elaborate compensation would not have negated the need of machining operations. Considering that the tolerance requirements for fitting the inserts into the die is in ISO class 6 with a very high surface smoothness $(\mathrm{Ra}<1 \mu \mathrm{m})$. This is not achievable with today's SLM printers. If the whole die had been printed, when it would not have been necessary to make the precision fitting, so the die would have been directly usable after printing and hardening. In addition, some light grinding of the die cavity and contact surfaces would have been necessary. This is how the injection mould in reference [14] was created.

In dies the material properties are important. No material testing has yet been performed on the printed dies. As an indication only slight visible porosity was seen on the machined surfaces. Closer inspection will be needed to determine the resulting properties. General expected material properties from the 1.2709 material is a yield strength of $900 \mathrm{MPa}$, UTS $1000 \mathrm{MPa}$ and an elongation to break of $6 \%$.

After printing with the SLM method, the cooling channels could be easily cleared of remaining powder using compressed air. However, the channels are moderately complex as seen in figure 3. In dies with more complex channels, this will be more difficult. Perhaps flexible hoses with specialized nozzles that can be used.

\section{Conclusions and future work}

The implications of manufacturing die inserts for HPDC have been highlighted. Extensive machining was needed to arrive at the required tolerance and surface smoothness. This adds to the cost and processing time of the die. The potential of the SLM method is not being fully utilized showing that effort must be made to bring down the amount of secondary operations. This can possible be achieved by redesigning the die assembly to bring down the tolerance requirements to levels that can be obtained as printed.

Future work involves casting fatigue test samples and examining the effect on the die. The actual mechanical properties of the inserts will be measured.

\section{Acknowledgement}

The KK-foundation and participating companies are greatly acknowledged for their financial support, research contributions and co-production in the project Distinct -Design Methods for Customized Products when Introducing Additive and Cyclic Manufacturing. The projects duration was between the years $2017-2020$. 


\section{References}

[1] Reggiani, B. and I. Todaro, Investigation on the design of a novel selective laser melted insert for extrusion dies with conformal cooling channels. International Journal of Advanced Manufacturing Technology, vol. 104, Issue (1-4), 2019, pp. 815-830.

[2] Booth, J.W., et al., The Design for Additive Manufacturing Worksheet. Journal of Mechanical Design, vol 139, issue 10, 2017, pp. 1-9.

[3] Boyer, J., et al., Special Section: Designing for additive manufacturing recent advances in design for additive manufacturing. Journal of Mechanical Design, Transactions of the ASME, vol. 139 issue 10,2017

[4] Hällgren, S., L. Pejryd, and J. Ekengren, (Re)Design for Additive Manufacturing. Procedia CIRP, Issue 50. (Supplement C), 2016, pp. 246-251.

[5] Klahn, C., B. Leutenecker, and M. Meboldt. Design strategies for the process of additive manufacturing. in Procedia CIRP, vol 36, 2015, pp 230-235.

[6] Leutenecker-Twelsiek, B., C. Klahn, and M. Meboldt, Considering Part Orientation in Design for Additive Manufacturing. Procedia CIRP, vol 50, 2016, pp. 408-413.

[7] Ranjan, R., R. Samant, and S. Anand, Integration of Design for Manufacturing Methods with Topology Optimization in Additive Manufacturing. Journal of Manufacturing Science and Engineering, Transactions of the ASME, vol. 139, issue 6, 2017.

[8] Reddy, S.N., et al. Application of topology optimization and design for additive manufacturing guidelines on an automotive component. in Proceedings of the ASME Design Engineering Technical Conference, vol 2A, 2016.

[9] Jahan, S.A. and H. El-Mounayri, Optimal Conformal Cooling Channels in 3D Printed Dies for Plastic Injection Molding. Procedia Manufacturing, vol 5, pp. 888-900, 2016.

[10] Kuo, C.C. and M.R. Li, Development of sheet metal forming dies with excellent mechanical properties using additive manufacturing and rapid tooling technologies. International Journal of Advanced Manufacturing Technology, vol 90, issue (1-4), 2017, pp. 21-25.

[11] Mazur, M., et al., Numerical and experimental evaluation of a conformally cooled H13 steel injection mould manufactured with selective laser melting. International Journal of Advanced Manufacturing Technology, vol 93, issue(1-4), 2017, pp. 881-900.

[12] Phull, G.S., S. Kumar, and R.S. Walia, Conformal cooling for molds produced by additive manufacturing: A review. International Journal of Mechanical Engineering and Technology, vol, 9 issue 1, 2018, pp. 1162-1172.

[13] Tang, Y., Z. Gao, and Y.F. Zhao, Design of conformal porous structures for the cooling system of an injection mold fabricated by additive manufacturing process. Journal of Mechanical Design, Transactions of the ASME, 2019, vol 141, issue 10, 2019

[14] PLMGroup. 3DMetPrint / PlastSystem AB, 2019, Accessed: 29.11.2019 [Online]. Available: https://plmgroup.se/referenser/3dmetprint-plastsystem-ab/.

[15] Queudeville, Y., U. Vroomen, and A. Bührig-Polaczek, Modularization methodology for high pressure die casting dies. International Journal of Advanced Manufacturing Technology, vol 71, issue (9-12): 2014, pp. 1677-1686.

[16] R. Stolt, et al. , Challenges and Support when Introducing AM in HPDC Tooling, In: 26th ISTE International Conference on Transdisciplinary Engineering, Kashiwa Campus, Japan, 2019, pp. 147-156.

[17] Müller, S., et al., An Initial Study of a Lightweight Die Casting Die Using a Modular Design Approach, International Journal of Metalcasting, vol 12, issue 4, 2018, pp: 870-883. 